\title{
A comprehensive study of oxidative stress in patients with chronic otitis media
}

\author{
Mustafa Celik ${ }^{1}$, İsmail Koyuncu² \\ 1 Department of Otorhinolaryngology, Harran University Medical Faculty, Sanliurfa, Turkey \\ 2 Department of Biochemistry, Harran University Medical Faculty, Sanliurfa, Turkey \\ Mustafa Celik, ORCID: 0000-0002-6237-2518 \\ Ísmail Koyuncu, ORCID: 0000-0002-9469-4757
}

\begin{abstract}
Objective: The purpose of this research study was to evaluate dynamic thiol/disulphide homeostasis (TDH) as a novel oxidative stress marker in participants with noncholesteatomatous chronic otitis media (ncCOM) and cholesteatomatous chronic otitis media (cCOM), as well as to investigate lipid hydroperoxide ( $\mathrm{LOOH})$, total antioxidant status (TAS), total oxidant status (TOS) and oxidative stress index (OSI) markers and compare the results with a healthy control group.

Methods: This research was carried out at Harran University Medical Faculty Ear-Nose-Throat Department, between April 2017 and April 2019. This was a prospective controlled trial study including 121 participants, 40 ncCOM, 39 cCOM and 42 healthy controls. Total thiol, native thiol, disulphide levels and $\mathrm{LOOH}$, TAS, TOS levels were measured in plasma of all patients and healthy volunteers.
\end{abstract}

Results: In our study, we found that total thiol, native thiol levels, native thiol/total thiol ratios and TAS levels were significantly lower in CCOM patients compared with the control group $(p<0.01)$. Disulphide/native thiol, disulphide/total thiol ratios, LOOH, TOS and OSI levels were significantly increased in the cCOM group compared with the control group $(p<0.01)$.

Conclusion: Oxidative stress (OS) is believed to be one of the underlying reasons for COM pathogenesis. In this research, we analyzed dynamic TDH as a novel OS marker using a novel improved fully automatic colorimetric technique, along with other parameters. This study may shed light on understanding the underlying reasons for the pathogenesis of COM and identify potential targets for antioxidant drug treatment.

Keywords: Otitis media, oxidative stress, thiols, homeostasis. 


\section{Introduction}

Chronic otitis media (COM) is an inflammatory process in the middle ear that is characterized by tympanic membrane perforation, ear discharge and hearing loss, lasting longer than 3 months and not completely resolving with medical treatment. ${ }^{[1]}$ It can be divided into two groups: noncholesteatomatous COM (ncCOM) and cholesteatomatous $\mathrm{COM}(\mathrm{cCOM})$. Cholesteatoma is an accumulation in the middle ear, where there is no keratinizing squamous epithelium, or in other pneumatized regions of the temporal bone. Cholesteatoma is a histologically benign but clinically destructive lesion. Despite numerous studies on how the keratinizing squamous epithelium reaches the middle ear and how it begins hyperkeratinization, the etiopathogenesis has not yet been fully elucidated. ${ }^{[2]}$

Degradation of the balance between free radicals in biological systems and antioxidants with its sweeping effect is known as oxidative stress (OS). ${ }^{[3,4]}$ According to reports, increased reactive oxygen species (ROS) levels may have an effect on the chronicity of middle ear inflammation and the etiopathogenesis of COM when oxidative stress increases or the antioxidant defense system has a deficiency. ${ }^{[4,5]}$

Thiols are organic compounds that contain a sulfhydryl group $(-\mathrm{SH})$. They react with free radicals to inhibit cellular and tissue detriments that are stimulated by reactive oxygen agents. With the effect of OS, thiols are turned into disulphides. By recycling the emerging reversible disulphide species, thiols can be obtained. This cycle is described as TDH (thiol-disulphide homeostasis), which is an active condition functioning endlessly. This cycle plays a vital role in antioxidant defense mechanisms, oxidative stress, enzymatic processes, detoxification procedures and apoptosis. ${ }^{[6-8]} \mathrm{In}$ the year 2014, a new and automatic method that directly measured serum thiol/disulphide levels was developed by Erel and Neselioglu. ${ }^{[6]}$ There are other parameters such as total oxidant status (TOS), total antioxidant status (TAS), lipid hydroperoxide (LOOH) and oxidative stress index (OSI) that are used to calculate oxidant/antioxidant balance in pathophysiological disorders. ${ }^{[3,9]}$

In this study, we aimed to analyze the relationship between COM and dynamic TDH as a novel and unexplored oxidative stress marker, in addition to TAS, TOS, LOOH and OSI. This is an important and initial study that investigates the link between TDH and COM with other oxidative stress markers.

\section{Materials and Methods}

This was a prospective controlled study including cases with ncCOM (group 1) and cCOM (group 2) and a healthy control group (group 3). Group 1 and group 2 cases consisted of patients who were operated in our department. Local ethics committee permission was obtained from the Harran University (date: 11.05.2017;05/18) before starting the study. Consent forms were signed by all participants included in the study. The research procedures were consistent with the rules of the Helsinki Declaration.

A total of 121 patients consisting of $40 \mathrm{ncCOM}$ patients (group 1), 39 cCOM patients (group 2) and 42 healthy controls (group 3) were included in the research. The diagnosis of cholesteatoma was also confirmed pathologically. The healthy control group matched with both patient groups in terms of age, gender and body mass index (BMI) (Table 1).

A detailed history, basic otoscopic examination, audiological examination (pure tone audiometry) and microscopic ear examination were applied to the patient groups, as well as the control group. An evaluation was conducted using temporal bone tomography of the patients with cCOM. In this study, which included patients between 18 and 52 years of age, the main inclusion criterium was conduction hearing loss on audiometric examination. Exclusion criteria were acute exacerbations of COM, vestibular disorders, auditory tinnitus, sensorineural hearing loss, history of temporal

\begin{tabular}{|c|c|c|c|c|c|}
\hline Variable & Group 1 (n:40) & Group 2 (n:39) & Group 3 (n:42) & $\begin{array}{c}\text { (Group } 1 \text { vs } 3 \text { ) } \\
\text { p-value }\end{array}$ & $\begin{array}{c}\text { (Group } 2 \text { vs } 3 \text { ) } \\
\text { p-value }\end{array}$ \\
\hline Age (years) & $33.67 \pm 7.49$ & $31.49 \pm 7.51$ & $33.19 \pm 8.63$ & $>0.05$ & $>0.05$ \\
\hline Gender (Male/Female) & $23 / 17$ & $24 / 15$ & $26 / 16$ & $>0.05$ & $>0.05$ \\
\hline Body Mass Index $\left(\mathrm{kg} / \mathrm{m}^{2}\right)$ & $25.09 \pm 1.3$ & $25.33 \pm 1.29$ & $25.64 \pm 1.2$ & $=0.05$ & $>0.05$ \\
\hline
\end{tabular}


temporal bone trauma, history of neuro-otological surgery, history of ototoxic drug, chronic drug use and liver, renal, hematologic, cardiovascular, neurological or psychiatric illnesses and malignancies. Patients with the presence of one or more of the above-mentioned disorders were excluded from the study.

\section{Biochemical Analysis}

After approximately 8 hours of the fasting period, blood samples to be used for biochemical analysis were taken from peripheral veins. Without waiting, samples were centrifuged at $3000 \mathrm{x}$ g for 10 minutes and stored at -80 degrees until sufficient numbers were reached. To evaluate oxidative status, TDH markers, $\mathrm{LOOH}$, TOS, TAS and OSI values were analyzed in stored samples.

\section{TDH Analysis}

A new and fully automated technique defined by Erel et al, was used to measure total thiol, disulphide levels and native thiol in samples stored under appropriate conditions. ${ }^{[6]}$ This new method is based on the conversion of sulfhydryl groups of proteins into recycled disulphide forms under oxidative conditions and the reduction of disulphide compounds to thiol groups once the oxidative stress is eliminated. The amount of dynamic disulphide compounds was determined by dividing the difference of total thiol and native thiol values by two. Following this, total thiol and disulphide amounts, native thiol/total thiol ratios, disulphide/total thiol ratios and disulphide/native thiol ratios were calculated as a percentage.

\section{LOOH Analysis}

Serum $\mathrm{LOOH}$ values were determined using an automated xylenol orange method. ${ }^{[10,11]}$ Ferrous ions were obtained when LOOH was oxidized to ferric ions. The obtained results were evaluated using xylenol orange.

\section{TOS, TAS and OSI Analysis}

TOS values detected using the Rel Assay Diagnostics kit (Rel Assay Diagnostics kit, Mega Tip, Gaziantep, Turkey)

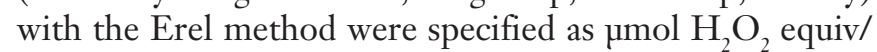
L. ${ }^{[12]}$ In the Erel method, oxidation reactions that are increased by glycerol molecules, oxidize ferrous ion-odianisidine complex to ferric ions. The color intensity of the iron ions resulting from the complex formed by the xylenol orange indicates the total amount of oxidants. Calibration of the test was performed using hydrogen peroxide. TAS values calculated using the Rel Assay Diagnostics kit (Rel Assay Diagnostics kit, Mega Tip, Gaziantep, Turkey) were specified as $\mu \mathrm{mol}$ Trolox Equiv/ L. ${ }^{[12]}$ The Fenton reaction induces free radical reactions and this was followed using dianisidyl radical absorbance. Free dianisidyl radicals were used in relative amounts in the measurement of the antioxidative effect. The formula OSI (arbitrary unit) $=$ TOS $(\mathrm{mmol} \mathrm{H} 2 \mathrm{O} 2$ equivalent/L) / TAS (mmol Trolox equivalent/L) was used to determine the OSI value. ${ }^{[12,13]}$

\section{Statistical Analysis}

SPSS software version 22.0 (SPSS Inc., Chicago, IL, USA) was used to evaluate the statistical analysis of all data. Pearson chi-squared test was performed in gender evaluation statistics of the groups. The obtained comparison results were reported as percentages and numbers. An independent sample t-test was used to compare continuous variables between the groups. The results of this comparison are given as mean (standard deviation). For values $<0.05, \mathrm{p}$ was accepted to be statistically significant.

\section{Results}

Age, gender and BMI data of all groups are presented in Table 1. Biochemical data of group 1, group 2 and group 3 are presented in Table 2.

\section{Discussion}

The main purpose of this study was to evaluate the relationship between COM and OS with LOOH, TAS, TOS and OSI parameters, in addition to TDH as a novel OS parameter. Previous studies have examined TOS, TAS and OSI values in cases with $\mathrm{COM}^{[4]}$ With regard to the literature, our current study uniquely differs from previous research since the TDH marker was analyzed for the first time in this research study. For this purpose, serum levels of $\mathrm{LOOH}$, TOS, TAS, OSI and also TDH parameters in patients were compared for the ncCOM, cCOM, and healthy control groups.

ROS may damage the integrity, structure and function of the cell membrane, proteins and deoxyribonucleic acid (DNA). ${ }^{[3,14]}$ OS is accepted to play an important role in the pathogenesis of several diseases prevalent in humans and experimental animals. ${ }^{[15-19]}$ Furthermore, LOOHs resulting from lipid peroxidation by ROS disrupt the integrity of the cell membrane. ${ }^{[18,19]}$ 
Table 2: Biochemical data of Group 1, Group 2 and Group 3

\begin{tabular}{|c|c|c|c|c|c|}
\hline Biochemical markers & $\begin{array}{c}\text { Group } 1 \\
(m e a n \pm S D)\end{array}$ & Group $2($ mean $\pm S D)$ & Group $3(\operatorname{mean} \pm S D)$ & $\begin{array}{l}\text { (Group 1-3) } \\
\text { p-value }\end{array}$ & $\begin{array}{l}\text { (Group 2-3) } \\
\text { p-value }\end{array}$ \\
\hline Native thiol $(\mathrm{mmol} / \mathrm{L})$ & $407.52 \pm 43.10$ & $378.22 \pm 50.22$ & $427.37 \pm 41.66$ & $<0.05$ & $<0.01$ \\
\hline Total thiol (mmol/L) & $439.15 \pm 37.03$ & $418.04 \pm 39.71$ & $456.24 \pm 36.73$ & $<0.05$ & $<0.01$ \\
\hline Disulphide (mmol/L) & $15.81 \pm 13.34$ & $19.91 \pm 14.50$ & $14.43 \pm 11.33$ & 0.69 & 0.06 \\
\hline Disulphide/native thiol (\%) & $4.12 \pm 3.93$ & $5.69 \pm 4.76$ & $3.54 \pm 3.21$ & 0.53 & $<0.01$ \\
\hline Disulphide/total thiol (\%) & $3.59 \pm 3.02$ & $4.82 \pm 3.48$ & $3.16 \pm 2.49$ & 0.55 & $<0.01$ \\
\hline Native thiol/total thiol (\%) & $92.8 \pm 6.04$ & $90.35 \pm 6.96$ & $93.66 \pm 4.99$ & 0.55 & $<0.01$ \\
\hline Lipid hydroperoxide ( $\mu \mathrm{mol} / \mathrm{L})$ & $9.2 \pm 1.39$ & $10.23 \pm 1.08$ & $8.95 \pm 1.15$ & 0.43 & $<0.01$ \\
\hline $\begin{array}{l}\text { Total antioxidant status } \\
\text { (mmol Trolox Equiv/L) }\end{array}$ & $1.14 \pm 0.28$ & $1 \pm 0.12$ & $1.15 \pm 0.23$ & 0.92 & $<0.01$ \\
\hline $\begin{array}{l}\text { Total oxidant status } \\
\text { ( } \mu \mathrm{mol} \mathrm{H}_{2} \mathrm{O}_{2} \text { equiv/L) }\end{array}$ & $13.07 \pm 1.75$ & $22.25 \pm 2.98$ & $11.10 \pm 0.93$ & $<0.01$ & $<0.01$ \\
\hline $\begin{array}{l}\text { Oxidative stress index } \\
\text { (arbitrary units ) }\end{array}$ & $1.19 \pm 0.29$ & $2.24 \pm 0.43$ & $1.00 \pm 0.20$ & $<0.01$ & $<0.01$ \\
\hline
\end{tabular}

Although many different factors have been blamed for the etiopathogenesis of COM, this has not been elucidated in all aspects. ${ }^{[2,20]}$ Due to oxidative stress, many factors such as genetics, eustachian tube dysfunction, autoimmunity, environmental factors, socioeconomic factors, recurrent upper respiratory tract infections, craniofacial malforma-tions, toxins, lipid peroxidation products, chemokines and cytokines responsible for the chronic state of inflammation are implicated in the etiopathogenesis of COM. ${ }^{[21]}$ All of these factors are considered to increase the amount of free oxygen radicals (FORs) that are at the root of many pathological disorders. ${ }^{[22]}$ It has been suggested that FORs may have a role in the etiology of COM. COM usually develops on the basis of recurrent acute attacks on COM with effusion (OME). ${ }^{[23]}$ In the chronic process of inflammation, redox activity increases with the effect of mediators such as chemokines and cytokines. OS starts and continues as a result of the stimulation of redox mechanisms.

Peroxidation of lipids in middle ear cells can delay the healing process and increase inflammation severity. This process can eventually lead to a chronic condition. Studies have shown that ROS may be effective in the chronic process of otitis media with effusion. ${ }^{[24]}$ Yilmaz et al [25] have examined the effects of ventilation tube application on the oxidant/antioxidant balance of children with EOM. In this research, they reported that the plasma antioxidant level increases and the oxidant level decreases in children with EOM after surgery. Khakimov et al [26] reported thatthe oxidation products of children with suppurative otitis media had higher levels than the control group and lower antioxidant enzyme levels. Increased chronic inflammation in the middle ear mucosa may lead to polyp and cholesteatoma development due to mucosal metaplasia. ${ }^{[27]}$ Hamzei $M$ et al ${ }^{[28]}$ have shown that activation of macrophages and T-cells is elevated in the cholesteatoma tissue. Another study suggests that there is a relationship between chronic inflammation and bacterial biofilms in the cholesteatoma caused by pathological activation of osteoclasts through proinflammatory cytokines. ${ }^{[29]}$ Camps et al ${ }^{[30]}$ suggested that antioxidant systems may be an effective protection system against the harmful effects of biofilms. In another study, it was suggested that there is a statistically significant reduction in the antioxidant system in cases with $\mathrm{CCOM}$, and this decrease may have a major part in biofilm development in the tissue of cholesteatoma. ${ }^{[4]}$ In our study, the detection of more pronounced oxidative stress findings in $\mathrm{CCOM}$ supports this view.

TDH is an important component of the basic defensive systems of the body against the detrimental effects of OS. As a result of $\mathrm{TDH}$ deterioration, disulphide compounds that are continuously increasing may lead to various disorders in many systems of the human body. Therefore, the importance of $\mathrm{TDH}$ is increasing more and more. 
Our study is the first research study in the literature to investigate $\mathrm{TDH}$, a new parameter of $\mathrm{OS}$, along with other oxidative stress markers in COM pathogenesis. The main limitations of the present study were the low number of samples and the absence of tissue work.

\section{Conclusion}

The data obtained from this research study demonstrate that there is a process by which OS plays a role at the beginning and in the continuation of COM and that cases with CCOM are exposed to strong OS. Despite the immense amount of research on the mechanisms of action of ROS on cellular structures, it continues to be an intriguing field since it relates to the pathogenesis of several diseases. We think that new and more detailed studies in this field will play a significant $r$ ole in describing the etiopathogenesis of diseases in the future and the development of new treatment protocols.

\section{Acknowledgements: None}

\section{References}

1. Goycoolea MV, Hueb MM, Ruah C. Otitis media: the pathogenesis approach. Definitions and terminology. Otolaryngol Clin North Am 1991;24:757-61.

2. Kemppainen HO, Puhakka HJ, Laippala PJ, Sipila MM, Manninen MP, Karma PH. Epidemiology and aetiology of middle ear cholesteatoma. Acta Otolaryngol 1999;119:568-72.

3. Celik M, Koyuncu İ. A Comprehensive Study of Oxidative Stress in Tinnitus Patients. Indian J Otolaryngol Head Neck Surg 2018;70:521-6.

4. Baysal E, Aksoy N, Kara F, et al. Oxidative stress in chronic otitis media. Eur Arch Otorhinolaryngol 2013;270:1203-8.

5. Yariktas M, Doner F, Dogru H, Yasan H, Delibas N. The role of free oxygen radicals on the development of otitis media with effusion. Int $\mathrm{J}$ Pediatr Otorhinolaryngol 2004;68:889-94.

6. Erel O, Neselioglu S. A novel and automated assay for thiol/disulphide homeostasis. Clin Biochem 2014;47:326-32.

7. Cremers CM, Jakob U. Oxidant sensing by reversible disulfide bond formation. J Biol Chem 2013;288:26489-96.

8. Celik M, Koyuncu İ. Oxidative stress in prelingual sensorineural hearing loss and the effects of cochlear implant application on serum oxidative stress levels. Int J Pediatr Otorhinolaryngol 2019;119:177-82.

9. Girotti AW. Lipid hydroperoxide generation, turnover, and effector action in biological systems. J Lipid Res 1998;39:1529-42.

10. Erel O. Automated measurement of serum ferroxidase activity. Clin Chem 1998;44:2313-9.

11. Nourooz-Zadeh J. Ferrous ion oxidation in presence of xylenol orange for detection of lipid hydroperoxides in plasma. Methods Enzymol 1999;300:58-62.
Ethics Committee Approval: The study was approved by the local ethics committee of the Harran University Faculty of Medicine (11.05.2017; 05/18).

Informed Consent: All participants provided written informed consent to the study.

Author Contributions: Designing the study - M.Ç., İ.K.; Collecting the data - M.Ç., İ.K.; Analyzing the data - M.Ç., İ.K.; Writing the manuscript - M.Ç., İ.K.; Confirming the accuracy of the data and the analyses - M.Ç., IK..

Conflict of Interest: The authors have no conflicts of interest to declare.

Financial Disclosure: The authors declare that this study has received no financial support.

12. Erel O. A new automated colorimetric method for measuring total oxidant status. Clin Biochem 2005;38:1103-11.

13. Erel O. A novel automated method to measure total antioxidant response against potent free radical reactions. Clin Biochem 2004;37:112-9.

14. Gupta RK, Patel AK, Shah N, et al. Oxidative stress and antioxidants in disease and cancer: a review. Asian Pac J Cancer Prev 2014;15:4405-9.

15. Ates I, Kaplan M, Yuksel M, et al. Determination of thiol/disulphide homeostasis in type 1 diabetes mellitus and the factors associated with thiol oxidation. Endocrine 2016;51:47-51.

16. Yuksel M, Ates I, Kaplan M, et al. The dynamic thiol/disulphide homeostasis in inflammatory bowel disease and its relation with disease activity and pathogenesis. Int J Colorectal Dis 2016;31:1229-31.

17. Şimşek E, Erel O, Bicer CK, Çarlıoğlu A. A novel method for determining the relation between nasal polyposis and oxidative stress: the thiol/ disulphide homeostasis. Acta Otolaryngol 2016;136:1180-3.

18. Cross CE, Halliwell B, Borish ET, et al. Oxygen radicals and human disease. Ann Intern Med 1987;107:526-45.

19. Porter NA, Mills KA, Caldwell SE. Mechanisms of free radical oxidation of unsaturated lipids. Lipids 1995;30:277-90.

20. Semaan MT, Megerian CA. The pathophysiology of cholesteatoma. Otolaryngol Clin North Am 2006;39:1143-59.

21. Karlidag T, Ilhan N, Kaygusuz I, Keles E, Yalcin S. Comparison of free radicals and antioxidant enzymes in chronic otitis media with and without tympanosclerosis. Laryngoscope 2004;114:85-9.

22. Valko M, Leibfritz D, Moncol J, Cronin MT, Mazur M, Telser J. Free radicals and antioxidants in normal physiological functions and human disease. Int J Biochem Cell Biol 2007;39:44-84. 
23. Tos M. Sequelae of secretory otitis media and the relationship to chronic suppurative otitis media. Ann Otol Rhinol Laryngol 1990;99:18-9.

24. Döner F, Delibas N, Dogru H, Yariktas M, Demirci M. The role of free oxygen radicals in experimental otitis media. J Basic Clin Physiol Pharmacol 2002;13:33-40.

25. Yılmaz T, Koçan EG, Besler HT, Yılmaz G, Gürsel B. The role of oxidants and antioxidants in otitis media with effusion in children. Otolaryngol Head Neck Surg 2004;131:797-803.

26. Khakimov AM, Arifov SS, Faizulaeva FN. Efficacy of antioxidant therapy in patients with acute and chronic purulent otitis media. Vestn Otorinolaringol 1997;5:16-9.
27. Bernstein V. Middle ear mucosa histological, histochemical immunochemical and immunologic aspects. In: John AF, Sontos-Sacchi J, eds. Physiology of the ear. New York, NY: Raven Press; 1988. p. 59-80.

28. Hamzei M, Ventriglia G, Hagnia M, et al. Osteoclast stimulating and differentiating factors in human cholesteatoma. Laryngoscope 2003;113:436-42

29. Jung JY, Pashia ME, Nishimoto SY, Faddis BT, Chole RA. A possible role for nitric oxide in osteoclastogenesis associated with cholesteatoma. Otol Neurotol 2004;25:661-8.

30. Camps J, Pujol I, Ballester F, Joven J, Simo' JM. Paraoxonases as potential antibiofilm agents: their relationship with quorum-sensing signals in gram-negative bacteria. Antimicrob Agents Chemother 2011;55:132531.

This is an open access article distributed under the terms of the Creative Commons Attribution-NonCommercial-NoDerivs 3.0 Unported (CC BY- NC-ND3.0) Licence (http://creativecommons.org/licenses/by-nc-nd/3.0/) which permits unrestricted noncommercial use, distribution, and reproduction in any medium, provided the original work is properly cited.

Please cite this article as: Celik M, Koyuncu İ. A comprehensive study of oxidative stress in patients with chronic otitis media. ENT Updates 2020;10(1):238-243. 